\title{
Sum-Rate Performance of A NOMA-based Two-Way Relay Approach for A Two-User Cellular Network
}

\author{
Guosheng Li* \\ School of Intelligent Information Engineering, Zhejiang WanliUniversity \\ Ningbo, 315100, China \\ [e-mail: nblgs@foxmail.com] \\ *Correspondingauthor:Guosheng Li
}

Received January 22, 2021; revised March 21, 2021; accepted April 15, 2021; published May 31, 2021

\begin{abstract}
This paper considers a cellular two-way relay network with one base station (BS), one relay station (RS), and two users. The two users are far from the BS and no direct links exist, and the two users exchange messages with the BS via the RS. A non-orthogonal multiple access (NOMA) and network coding (NC)-based decode-and-forward (DF) two-way relaying (TWR) scheme TWR-NOMA-NC is proposed, which is able to reduce the number of channel-uses to three from four in conventional time-division multiple access (TDMA) based TWR approaches. The achievable sum-rate performance of the proposed approach is analyzed, and a closed-form expression for the sum-rate upper bound is derived. Numerical results show that the analytical sum-rate upper bound is tight, and the proposed TWR-NOMA-NC scheme significantly outperforms the TDMA-based TWR and NOMA-based one-way relaying counterparts.
\end{abstract}

Keywords: cellular two-way relay, NOMA, decode-and-forward, sum-rate performance 


\section{Introduction}

$\mathbf{N}_{\text {on-orthogonal multiple access (NOMA) is a promising solution to improve the spectral and }}$ energy efficiency of wireless communication networks [1][2]. By allowing multiple wireless terminals share the same radio resources, NOMA significantly improves transmission efficiency as compared with conventional time-division multiple access (TDMA) or frequency-division multiple access (FDMA) schemes.

The NOMA technique has been recently applied in wireless relay networks to further improve the sum-rates and the outage performance [3]-[6]. The authors in [3] proposed a three-user cooperative NOMA scheme, where the two near users with better link quality acts as relays to forward the signals to the far user. In [4], Yue et al, considered a two-user NOMA system, where the strong user helps the far user by decoding and forwarding the received NOMA signal. In their scheme, the strong user switches between full-duplex (FD) and half-duplex (HD) mode to improve the data rate, which is difficult to implement as designing such small-size FD mobile device is challenging. For wireless networks with dedicated relays, the authors in [5][6] proposed NOMA-based relaying schemes that the relay node and the direct link user forms a NOMA pair, and the relay forwards signal to the indirect link user with DF [5] or amplify-and-forward (AF) [6] relaying.

All these aforementioned NOMA-based relaying schemes involve one-way relaying only, i.e., the NOMA signal is transmitted from the source to the destination via the relay. The combination of NOMA and two-way relaying (TWR) was studied recently in [7]-[12]. In [9], the authors investigated a two-group NOMA TWR system, where the users are divided into two groups, and exchange data with one relay. In [10], a hybrid two-way relaying scheme was proposed, which combined the NOMA and network coding techniques. The authors in [11] investigated a NOMATWR network (TWRN) with multiple relays. It was shown in [11] that the spectral efficiency and the spatial diversity can be enhanced with NOMA transmission and relay selection.

Note that all these works [7]-[10] considered the classic two-way relay network model, i.e., two users exchange messages with the help of relay nodes. For more sophisticated two-way relaying systems, such as multipair TWRN [13-15] and multi-way relay network (MWRN) [16][17], the system throughput can also be improved by applying NOMA. In [13], the authors considered a multipair TWRN with NOMA transmission, in which multiple pairs of users communicate with each other with the help of several relay nodes. A rate splitting scheme with successive decoding was proposed to combat the interference. The authors in [14] proposed a cognitive radio inspired NOMA-based network coding scheme to improve the spectral efficiency of multipair TWRNs. In [15], Zhang and Jia designed beamforming matrices for performance optimization in a NOMA-based multi-pair TWRN. In [17], the authors investigated an unmanned aerial vehicle (UAV)-aided NOMA MWRN. Numerical results showed that these proposed approaches [15]-[17] can significantly improve the sum-rates.

In this work, we consider another important two-way relaying model, namely the cellular two-way relay network (cTWRN), which consists of a base station (BS), multiple mobile users and relay stations (RSs) [18]-[22]. For such a cTWRN as shown in Fig. 1, there exist four data streams in each round of transmission, i.e., two downlink data streams from the BS to the two users via the RS, and two uplink data streams from the two users to the BS via the RS. Existing schemes, such as those in [23][24], requires at least four time slots to complete each round of transmission due to the half-duplex mode of the relay. 
To reduce the number of required time slots, this paper proposes a TWR-NOMA-NC approach based on NOMA, network coding (NC), and TWR DF relaying. In TWR-NOMA-NC, only three time slots are needed for the two users to exchange data with the BS, as shown in Fig. 1(b). In the proposed scheme, an uplink NOMA is constructed at the relay in the first slot, and network-encoded signals are constructed in the second and third time slots. The main contributions of the paper are summarized as follows.

- A new NOMA and NC-based TWR scheme with DF relaying is proposed, which require only three time slots to exchange data between the BS and two distant users, substantially improving spectral utilization and efficiency as compared with existing schemes.

- The achievable sum-rate of the proposed TWR-NOMA-NC scheme is analyzed. Based on a SINR upper bound, a closed-from upper bound is derived for the ergodic sum-rate of the proposed TWR-NOMA-NC. The upper bound is shown to be tight.

- Numerical results show that the proposed TWR-NOMA-NC scheme significantly outperforms conventional TDMA-based TWR transmission approach and NOMA-based one-way relaying approach.

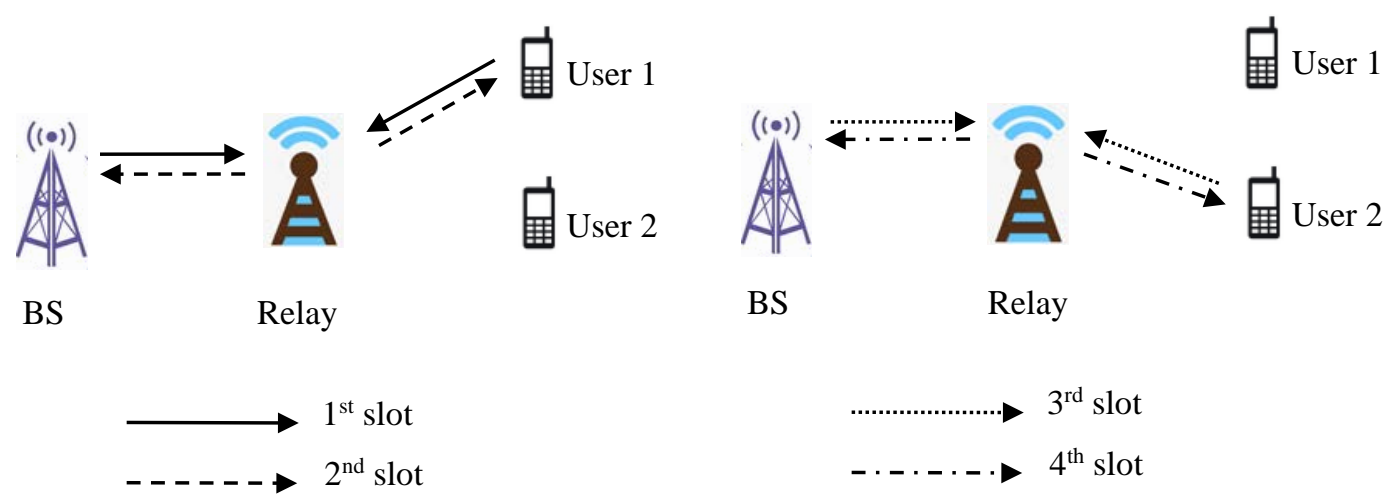

(a) Conventional TDMA-based scheme

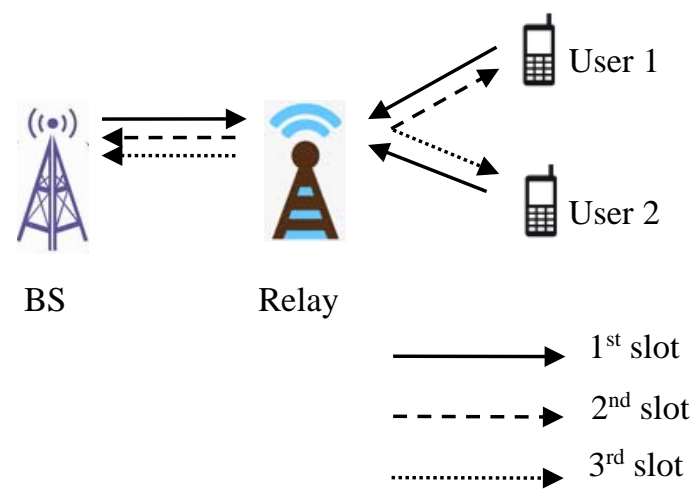

(b) The proposed scheme

Fig. 1. Conventional TDMA-based scheme and the proposed TWR-NOMA-NC scheme.

\section{The Proposed TWR-NOMA-NC Scheme}

Fig. 1 shows a two-user one-relay cTWRN, where a BS and two users $U_{k}, k=1,2$, exchange messages with a relay. All the nodes are half-duplex and the time-division duplex 
(TDD) mode is adopted. There are no direct links between the two users $U_{k}, k=1,2$, and the BS due to shadowing or deep fading. The channels are assumed to be reciprocal, Rayleigh flat fading. It is assumed that the channels remain unchanged during each round of transmission. The channel between the BS and the relay, and that between user $U_{k}$ and the relay are denoted by $h_{B R}$ and $h_{U R, k}, k=1,2$, respectively.We also assume that the BS and the relay have perfect knowledge of all the channels as assumed in [18]-[23]. Note that in practical systems, the BS can obtain the channel state information between the relay and the two users via channel feedback from the relay.

For conventional TDMA-based transmissions, four time slots are usually required for such a two-user cTWRN. That is, the BS exchanges messages with $U_{1}$ via the relayin the first and second time slots, while in the third and fourth time slots, the BS exchanges messages with $U_{2}$ via the help of the relay. To improve the spectral efficiency, this paper proposes a NOMA-based TWR-NOMA-NC scheme that can reduce the number of required time slots into three. Specifically, in the first slot, the BS and the two users send signals to the relay simultaneously to construct an uplink NOMA. The relay broadcasts the network-encoded signal to the BS and $U_{1}$ in the second slot. While in the third time slot, the relay broadcasts the network-encoded signal to the BS and $U_{2}$.

\subsection{The First Slot}

At the BS, let $s_{B, k}, k=1,2$, be the data symbol for user $U_{k}$ with a data rate of $R_{D L, k}$. The two information streams $s_{B, 1}$ and $s_{B, 2}$ are jointly encoded into a single data stream $s_{B}$. $s_{B}$ is then transmitted to the relay with a power of $P_{B}$. Meanwhile, user $U_{k}$ transmits $x_{U, k}=$ $\sqrt{P_{U, k}} s_{U, k}$, to the relay, where $s_{U, k}$ is the symbol at $U_{k}$, and $P_{U, k}$ is the power budget.

In the first slot, the relay's received signal is given by

where $z_{R} \sim C N\left(0, \sigma^{2}\right)$ is the noise.

$$
y_{R}=h_{B R} \sqrt{P_{B}} s_{B}+\sum_{k=1}^{2} h_{U R, k} \sqrt{P_{U, k}} s_{U, k}+z_{R} .
$$

From (1), an uplink NOMA is constructed at the relay in the first time slot. As the transmit power of the BS $P_{B}$ is usually much higher than the users, the relay detects the signal $s_{B}$ from the BS first, by treating the signals from the users as noises. Then, $s_{B}$ is removed from $y_{R}$ to detect $s_{U, 1}$ by treating $s_{U, 2}$ as interference, and $s_{U, 2}$ is detected at last. Here, it is assumed that user $U_{1}$ is the strong user with a better channel quality. Hence, $s_{U, 1}$ is decoded before detecting $s_{U, 2}$.

From (1), in the first slot, the transmission rate from the BS to the relay is given by

$$
R_{B R}=\frac{1}{3} \log _{2}\left(1+\frac{\left|h_{B R}\right|^{2} P_{B}}{\sum_{k=1}^{2}\left|h_{U R, k}\right|^{2} P_{U, k}+\sigma^{2}}\right) .
$$

After $s_{B}$ is successfully decoded, the signal $s_{B}$ is removed from $y_{R}$ to obtain

$$
y_{R, 1}=y_{R}-h_{B R} \sqrt{P_{B}} s_{B}=\sum_{k=1}^{2} h_{U R, k} \sqrt{P_{U, k}} s_{U, k}+z_{R} .
$$

Note that the relay detects $s_{U, 1}$ by treating $s_{U, 2}$ as interference in (3), and $s_{U, 2}$ is detected at last. From (3), the data rate from $U_{1}$ to the relay is

while that from $U_{2}$ to the relay can be expressed as

$$
R_{U R, 1}=\frac{1}{3} \log _{2}\left(1+\frac{\left|h_{U R, 1}\right|^{2} P_{U, 1}}{\left|h_{U R, 2}\right|^{2} P_{U, 2}+\sigma^{2}}\right)
$$

$$
R_{U R, 2}=\frac{1}{3} \log _{2}\left(1+\frac{\left|h_{U R, 2}\right|^{2} P_{U, 2}}{\sigma^{2}}\right) .
$$




\subsection{The Second Slot}

In the second slot, the relay performs XOR network coding as: $s_{R, 1}=s_{B, 1} \oplus s_{U, 1}$. The transmit signal of the relay is generated as $x_{R, 1}=\sqrt{P_{R}} s_{R, 1}$, where $P_{R}$ is the transmit power budget of the relay. $x_{R, 1}$ is then broadcasted to user $U_{1}$ andthe BS.

The received signal at the BS and user $U_{1}$ in the second slot are respectively given by

and

$$
y_{B, 1}=h_{B R} \sqrt{P_{R}} s_{R, 1}+z_{B, 1} \text {, }
$$

$$
y_{U, 1}=h_{U R, 1} \sqrt{P_{R}} s_{R, 1}+z_{U, 1}
$$

where $z_{B, 1} \sim C N\left(0, \sigma^{2}\right)$ and $z_{U, 1} \sim C N\left(0, \sigma^{2}\right)$ are the AWGNs.

From (7), both the BS and user $U_{1}$ are able to detect the desired signals directly. The achievable data rates from the relay to the BS, and that from the relay to user $U_{1}$ are given by

and

$$
R_{R B, 1}=\frac{1}{3} \log _{2}\left(1+\frac{\left|h_{B R}\right|^{2} P_{R}}{\sigma^{2}}\right)
$$

respectively.

$$
R_{R U, 1}=\frac{1}{3} \log _{2}\left(1+\frac{\left|h_{U R, 1}\right|^{2} P_{R}}{\sigma^{2}}\right)
$$

\subsection{The Third Slot}

The signal transmission and reception in the third slot is analogous with that in Section 2.2. The relay first performs XOR network coding and then forward signal $x_{R, 2}=\sqrt{P_{R}} s_{R, 2}$ to user $U_{2}$ and the BS, where $s_{R, 2}=s_{B, 2} \oplus s_{U, 2}$ is the network-encoded signal.

The received signals at the BS and users in the third slot are receptively given by

and

$$
y_{B, 2}=h_{B R} \sqrt{P_{R}} s_{R, 2}+z_{B, 2}
$$

$$
y_{U, 2}=h_{U R, 2} \sqrt{P_{R}} s_{R, 2}+z_{U, 2},
$$

where $z_{B, 2} \sim C N\left(0, \sigma^{2}\right)$ and $z_{U, 2} \sim C N\left(0, \sigma^{2}\right)$ are the AWGNs.

The data rates from the relay to the BS and user $U_{2}$ are given by

and

$$
R_{R B, 2}=\frac{1}{3} \log _{2}\left(1+\frac{\left|h_{B R}\right|^{2} P_{R}}{\sigma^{2}}\right),
$$

respectively.

$$
R_{R U, 2}=\frac{1}{3} \log _{2}\left(1+\frac{\left|h_{U R, 2}\right|^{2} P_{R}}{\sigma^{2}}\right)
$$

\section{Sum-Rate Performance}

\subsection{Achievable Sum-Rate}

Let $R_{D L, k}$ and $R_{U L, k}$ denote the downlink and uplink data rates of user $U_{k}, k=1,2$, respectively. For downlink transmission, from (2), for the link from the BS to the relay, $R_{D L, 1}$ and $R_{D L, 2}$ should satisfy:

$$
R_{D L, 1}+R_{D L, 2} \leq R_{B R}
$$

On the other hand, the downlink rates should not exceed that of the relay-user links in (9) and (13), i.e.,

$$
R_{D L, 1} \leq R_{R U, 1}, R_{D L, 2} \leq R_{R U, 2}
$$


From (14) and (15), the achievable down link sum-rates of the proposed approach is given by

$$
R_{D L, 1}+R_{D L, 2}=\min \left(R_{B R}, R_{R U, 1}+R_{R U, 2}\right) .
$$

For uplink transmission, from (4), (5), (8) and (12), we have

and

$$
R_{U L, 1}=\min \left(R_{U R, 1}, R_{R B, 1}\right) \text {, }
$$

$$
R_{U L, 2}=\min \left(R_{U R, 2}, R_{R B, 2}\right) .
$$

The sum-rate of the TWR-NOMA-NC scheme is then given by

$$
R_{\text {sum }}=\sum_{k=1}^{2} \min \left(R_{U R, k}, R_{R B, k}\right)+\min \left(R_{B R}, R_{R U, 1}+R_{R U, 2}\right) .
$$

\subsection{Ergodic Upper Bound}

The exact ergodic sum-rate in (19) is quite difficult to analyze. In the following, we derive a sum-rate upper bound for TWR-NOMA-NC. Let $\rho_{B}=P_{B} / \sigma^{2}, \rho_{R}=P_{R} / \sigma^{2}$, and $\rho_{U, k}=P_{U, k} / \sigma^{2}$. The mean channel gains are denoted by $G_{B R}=E\left(\left|h_{B R}\right|^{2}\right)$, and $G_{U R, k}=$ $E\left(\left|h_{U R, k}\right|^{2}\right), k=1,2$, respectively. We have the following result.

Theorem 1: The ergodic sum-rate of the proposed TWR-NOMA-NC scheme is upper bounded by

$$
\begin{aligned}
E\left(R_{\text {sum }}\right) \leq E\left(R_{\text {Sum }}^{U B}\right) & =\frac{1}{3 \ln 2\left(1-c_{1}\right)}\left[\Theta\left(c_{2}\right)-\Theta\left(c_{2} / c_{1}\right)\right]+\frac{1}{3 \ln 2} \Theta\left(c_{3}\right) \\
& +\sum_{k=1}^{2} \frac{1}{3 \ln 2} \Theta\left(\frac{1}{\rho_{R} G_{U R, k}},\right)
\end{aligned}
$$

where $E(\cdot)$ denote the expectation, $\Theta(x)=e^{x} E_{1}(x), E_{1}(x)$ is the exponential integral function, $c_{1}=\rho_{U, 2} G_{U R, 2} / \rho_{U, 1} G_{U R, 1}, c_{2}=\frac{1}{\rho_{U, 1} G_{U R, 1}}+\frac{1}{\rho_{R} G_{B R}}$, and $c_{3}=\frac{1}{\rho_{U, 2} G_{U R, 2}}+\frac{1}{\rho_{R} G_{B R}}$.

Proof: Using the inequality that $\min (X, Y) \leq Y$, we have $\min \left(R_{B R}, R_{R U, 1}+R_{R U, 2}\right) \leq$ $R_{R U, 1}+R_{R U, 2}$. Then, $R_{\text {sum }}$ in (19) can be upper bounded by

$$
R_{\text {sum }} \leq R_{\text {sum }}^{U B}=\sum_{k=1}^{2} \min \left(R_{U R, k}, R_{R B, k}\right)+R_{R U, 1}+R_{R U, 2} \text {. }
$$

Note that $R_{R U, k}=\frac{1}{3} \log _{2}\left(1+\gamma_{R U, k}\right), k=1,2$, where

$$
\gamma_{R U, k}=\left|h_{U R, k}\right|^{2} \rho_{R},
$$

whose cumulative distribution function (CDF) is given by

$$
F_{\gamma_{R U, k}}(x)=1-e^{-\rho_{R} G_{U R, k} x} \text {. }
$$

Then, the ergodic downlink rate of $U_{k}$ is given by

$$
\begin{aligned}
E\left(R_{R U, k}\right) & =\int_{0}^{+\infty} \frac{1}{3} \log _{2}(1+x) f_{\gamma_{R U, k}}(x) d x \\
& =\frac{1}{3 \ln 2} \int_{0}^{+\infty} \frac{1-F_{\gamma_{R U, k}}(x)}{1+x} d x \\
& =\frac{1}{3 \ln 2} \int_{0}^{+\infty} \frac{1}{1+x} e^{-\rho_{R} G_{U R, k} x} d x \\
& =\frac{1}{3 \ln 2} \Theta\left(\frac{1}{G_{U R, k} \rho_{R}}\right),
\end{aligned}
$$

where $\Theta(x)=e^{x} E_{1}(x), E_{1}(x)$ is the exponential integral function, and the last step in (24) is based on the equation $\int_{0}^{+\infty} e^{-a x} /(b+x) d x=e^{a b} E_{1}(a b)$ (cf. [25], eq.(5.1.28) ).

For uplink transmission of user $U_{1}$, the achievable rate can be expressed as

$$
\begin{aligned}
R_{U L, 1} & =\min \left(R_{U R, 1}, R_{R B, 1}\right) \\
& =\frac{1}{3} \log _{2}\left(1+\min \left(\gamma_{U R, 1}, \gamma_{R B, 1}\right)\right),
\end{aligned}
$$


where $\gamma_{U R, 1}=\frac{\rho_{U, 1}\left|h_{U R, 1}\right|^{2}}{1+\rho_{U, 2}\left|h_{U R, 2}\right|^{2}}$, and $\gamma_{R B, 1}=\rho_{R}\left|h_{B R}\right|^{2}$.

The CDF of $\gamma_{U R, 1}$ is given by

where $c_{1}=\rho_{U, 2} G_{U R, 2} / \rho_{U, 1} G_{U R, 1}$

$$
F_{\gamma_{U R, 1}}(x)=1-\frac{1}{1+c_{1} x} e^{-\frac{x}{\rho_{U, 1} G_{U R, 1}}},
$$

The CDF of $\gamma_{R B, 1}=\rho_{R}\left|h_{B R}\right|^{2}$ is

$$
F_{\gamma_{R B, 1}}(x)=1-e^{-\frac{x}{\rho_{R} G_{B R}}}
$$

Then, the CDF of $\gamma_{U L, 1}=\min \left(\gamma_{U R, 1}, \gamma_{R B, 1}\right)$ is given by

$$
\begin{aligned}
F_{\gamma_{U L, 1}}(x) & =1-P\left(\min \left(\gamma_{U R, 1}, \gamma_{R B, 1}\right) \leq x\right) \\
& =1-\left(1-F_{\gamma_{U R, 1}}(x)\right)\left(1-F_{\gamma_{R B, 1}}(x)\right) \\
& =1-\frac{1}{1+c_{1} x} e^{-c_{2} x},
\end{aligned}
$$

where $c_{2}=\frac{1}{\rho_{U, 1} G_{U R, 1}}+\frac{1}{\rho_{R} G_{B R}}$.

Based on (28), the ergodic uplink rate for user $U_{1}$ is given by

$$
\begin{aligned}
E\left(R_{U L, 1}\right) & =\int_{0}^{+\infty} \frac{1}{3} \log _{2}(1+x) f_{\gamma_{U L, 1}}(x) d x \\
& =\frac{1}{3 \ln 2} \int_{0}^{+\infty} \frac{1}{(1+x)\left(1+c_{1} x\right)} e^{-c_{2} x} d x \\
& =\frac{1}{3 \ln 2\left(1-c_{1}\right)}\left[\Theta\left(c_{2}\right)-\Theta\left(c_{2} / c_{1}\right)\right] .
\end{aligned}
$$

For uplink transmission of user $U_{2}$, the achievable rate can be expressed as

$$
\begin{aligned}
R_{U L, 2} & =\min \left(R_{U R, 2}, R_{R B, 2}\right) \\
& =\frac{1}{3} \log _{2}\left(1+\min \left(\gamma_{U R, 2}, \gamma_{R B, 2}\right)\right),
\end{aligned}
$$

where $\gamma_{U R, 2}=\rho_{U, 2}\left|h_{U R, 2}\right|^{2}, \gamma_{R B, 2}=\rho_{R}\left|h_{B R}\right|^{2}$.

The CDF of $\gamma_{U R, 2}$ is given by

While the CDF of $\gamma_{R B, 2}=\rho_{R}\left|h_{B R}\right|^{2}$ is

$$
F_{\gamma_{U R, 2}}(x)=1-e^{-\frac{x}{\rho_{U, 12 G_{U R, 2}}}} .
$$

$$
F_{\gamma_{R B, 2}}(x)=1-e^{-\frac{x}{\rho_{R} G_{B R}}} .
$$

Then, the CDF of $\gamma_{U L, 2}=\min \left(\gamma_{U R, 2}, \gamma_{R B, 2}\right)$ is given by

where $c_{3}=\frac{1}{\rho_{U, 2} G_{U R, 2}}+\frac{1}{\rho_{R} G_{B R}}$.

$$
F_{\gamma_{U L, 2}}(x)=1-e^{-c_{3} x} \text {, }
$$

Based on (33), the ergodic uplink rate for user $U_{2}$ is given by

$$
\begin{aligned}
E\left(R_{U L, 2}\right) & =\int_{0}^{+\infty} \frac{1}{3} \log _{2}(1+x) f_{\gamma_{U L, 2}}(x) d x \\
& =\frac{1}{3 \ln 2} \int_{0}^{+\infty} \frac{1}{1+x} e^{-c_{3} x} d x \\
& =\frac{1}{3 \ln 2} \Theta\left(c_{3}\right) .
\end{aligned}
$$

From (24), (29) and (34), we obtain the sum-rate upper bound in (20). 


\section{Computer Simulation}

In the simulation, the mean channel gains are set to be $G_{B R}=0 \mathrm{~dB}, G_{U R, 1}=-10 \mathrm{~dB}$ and $G_{U R, 2}=-20 \mathrm{~dB}$. The transmit power of the two users are the same: $P_{U, 1}=P_{U, 2}=P_{U}$, and the power budget of the $\mathrm{BS}$ is $30 \mathrm{~dB}$ higher than $P_{U}$, while $P_{R}$ is $10 \mathrm{~dB}$ higher than $P_{U}$. The noise variance is $\sigma^{2}=-20 \mathrm{dBm}$.

Fig. 2 shows the sum-rate of TWR-NOMA-NC under various SNRs, where the SNR in the figure is define as $S N R=P_{U} / \sigma^{2}$. The sum-rate bound in (20) is also shown in the figure. The sum-rate performance of the TDMA-based TWR NOMA scheme proposed in [23] (labeled as "TWR-NOMA scheme") and the NOMA-based one-way relaying scheme in [24] (labeled as "OWR-NOMA scheme") are also shown in the figure. Note that the OWR-NOMA scheme proposed in [24] was designed for the downlink transmission only. We extended this scheme to the considered cTWRN for two-way transmission. Specifically, in the first slot, the BS transmits to the relay, while the relay broadcasts NOMA signals to the two users in the second time slot. Similar uplink transmission takes place in the third and fourth time slots. From Fig. 2, it can be seen that the derived upper bound of TWR-NOMA-NC is tight, especially in the low-to-moderate SNR region. Compared with the conventional TWR-NOMA scheme and OWR-NOMA scheme, the proposed TWR-NOMA-NC scheme is able to achieve a much higher sum-rate in the whole SNR region. For instance, a sum-rate gain of $22 \%$ is obtained when $S N R=16 \mathrm{~dB}$ as compared with TWR-NOMA.

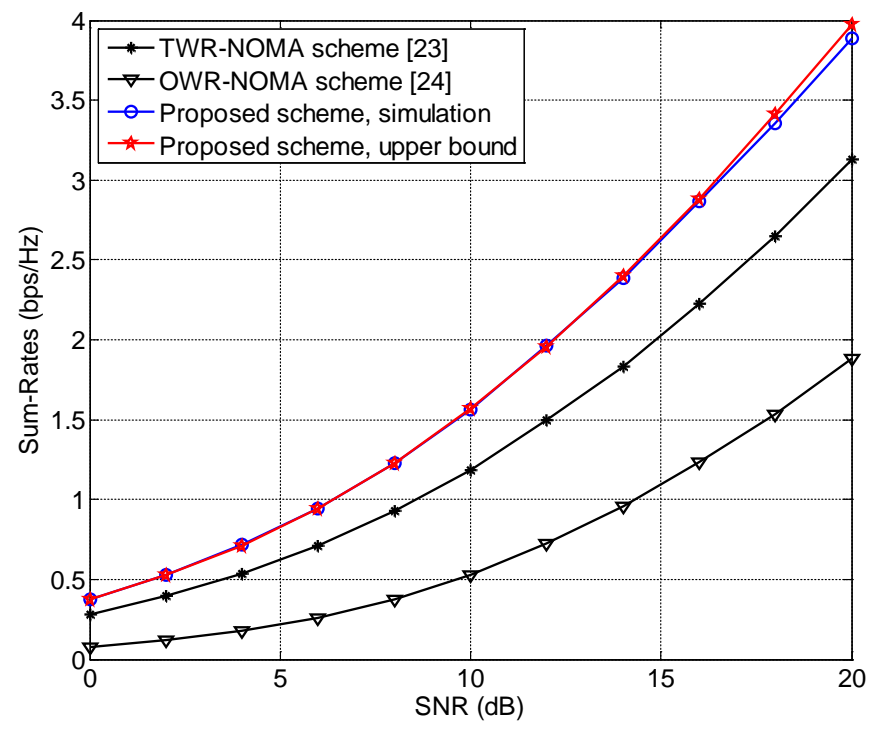

Fig. 2. Sum-rate performance of TWR-NOMA-NC.

The individual user rates of these three schemes are shown in Fig. 3. The simulation parameters are the same as in Fig. 2. For all these schemes, it can be seen that user $U_{1}$ is able to achieve a higher data rate and a lower outage probability, as user $U_{1}$ is the "strong" user with a better channel quality than user $U_{2}$. It is also shown that with TWR-NOMA-NC, both users are able to achieve a higher data rate than that of TWR-NOMA and OWR-NOMA schemes. Among all these three schemes, the OWR-NOMA scheme achieves the worst performance. This is due to the fact that in the downlink transmission in OWR-NOMA, the achievable data 
rate of user $U_{1}$ is limited by the power allocation factors at the BS.

The outage probability of the TWR-NOMA-NC scheme is shown in Fig. 4. The target downlink data rate is $0.5 \mathrm{bps} / \mathrm{Hz}$ for each user, while that of the uplink is $0.1 \mathrm{bps} / \mathrm{Hz}$. In Fig. 4, one can see that the proposed scheme outperforms TWR-NOMA and OWR-NOMA in terms of outage performance. For all these schemes, user $U_{1}$ achieves a lower outage probability than user $U_{2}$ since $U_{1}$ has a better channel condition than user $U_{2}$. For both TWR-NOMA-NC and TWR-NOMA schemes, the achievable data rate of user $U_{1}$ increases with the SNR, hence the corresponding outage probability decreases rapidly with the increased SNR. While for the OWR-NOMA scheme, the achievable data rates are limited by the power allocation factors. Hence, the outage probabilities of both users decrease slowly with the increased SNR.

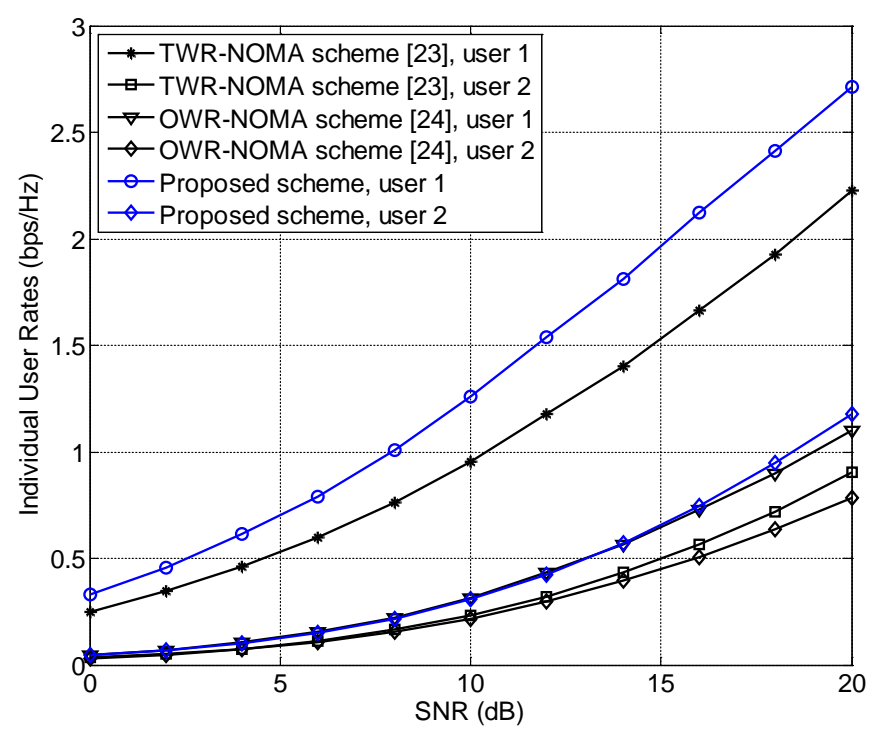

Fig. 3. Individual rate performance of TWR-NOMA-NC.

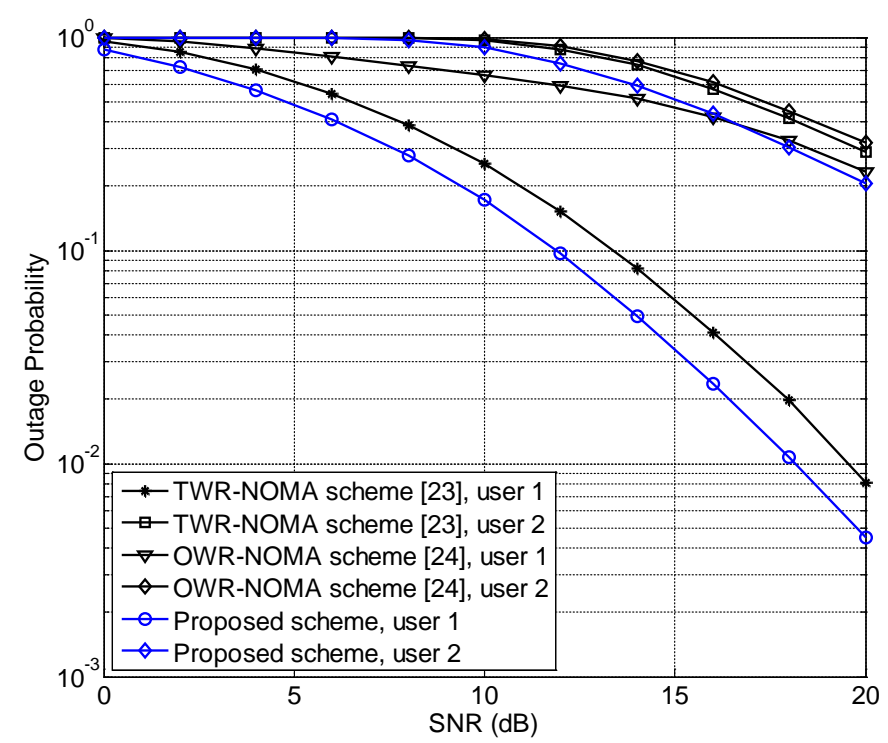

Fig. 4. Outage performance of TWR-NOMA-NC. 
Next, Fig. 5 investigates the sum-rate performance under different BS power budgets. The power of the relay is fixed to be $10 \mathrm{dBm}$, while those of the users are fixed to be $0 \mathrm{dBm}$ in the figure. From the figure, it can be seen that the sum-rate of TWR-NOMA-NC increases with the BS power, and outperforms the other two schemes. This is due to the fact that increasing the power of the BS can improve the downlink rates of both users in TWR-NOMA-NC. However, the sum-rates of all these schemes approach to certain constants for large $P_{B}$. This is due to the fact that for sufficient large BS power, the downlink rates are bottlenecked by the link from the relay to the users. Hence, the downlink rates approach to a constant as $P_{R}$ is fixed during the simulation.

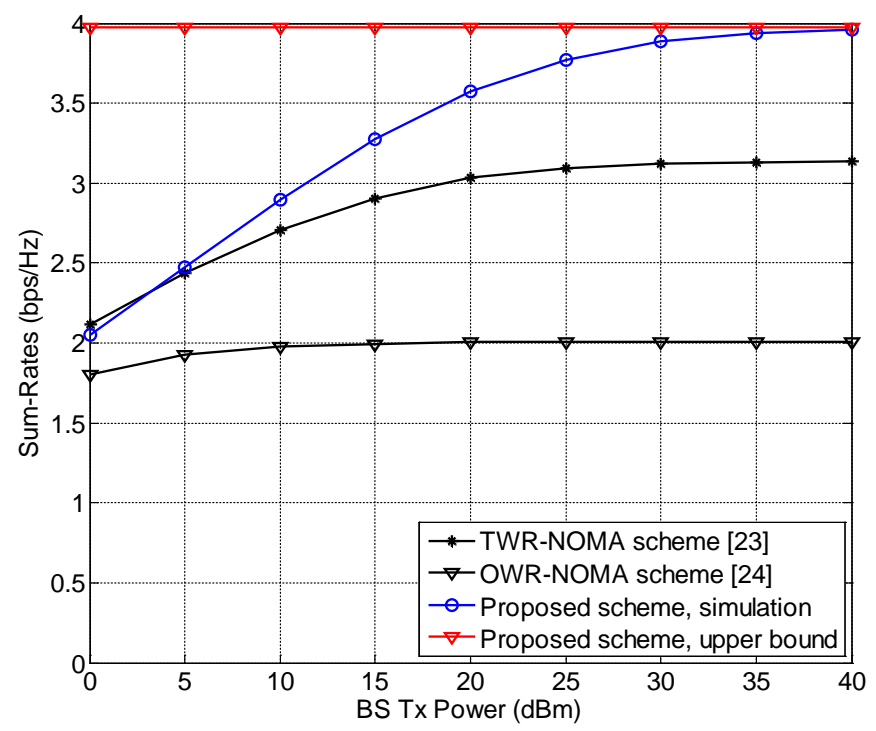

Fig. 5. Sum-rate vs BS power for TWR-NOMA-NC.

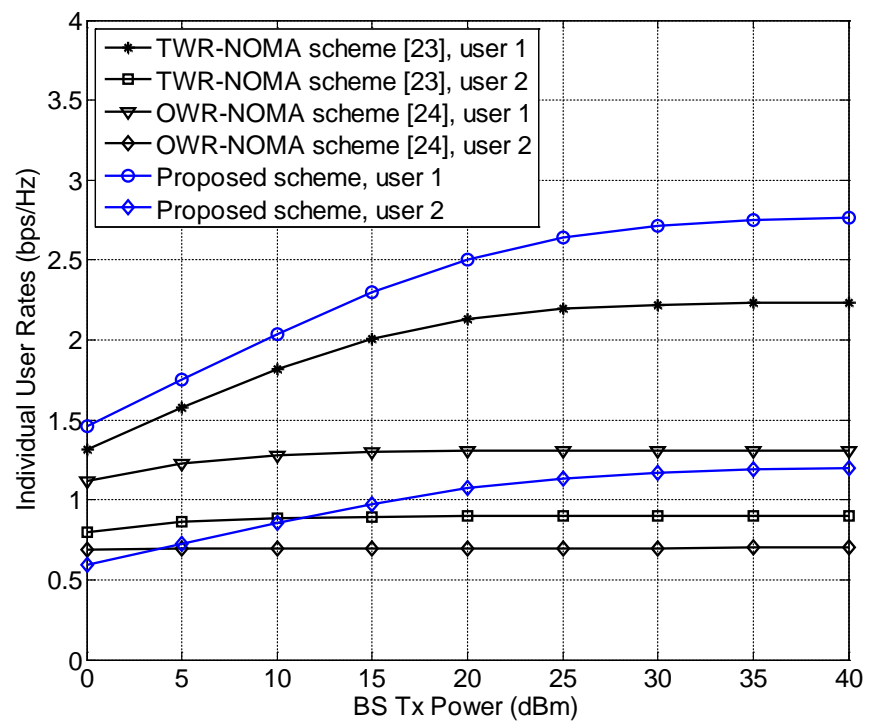

Fig. 6. Individual rate vs BS power for TWR-NOMA-NC. 


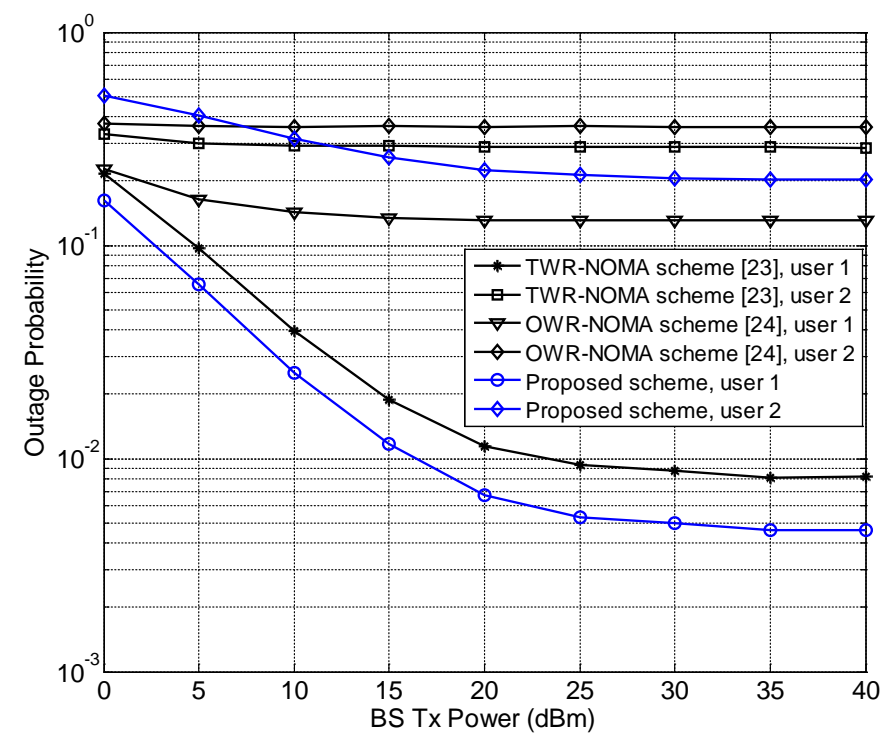

Fig. 7. Outage performance vs BS power for TWR-NOMA-NC.

Similar results can be observed for the individual rate performance shown in Fig. 6, where the achievable data rates of both users approach to certain constants for large BS power budgets. Again, the OWR-NOMA scheme achieves the lowest data rates among the three schemes. Especially for the strong user $U_{1}$, there is a large performance gap as compared to the other two schemes. There is little performance improvement for user $U_{1}$ in the OWR-NOMA scheme by increasing the transmit power of the BS. As a result, the OWR-NOMA scheme achieves a much lower sum-rate as shown in Fig. 5. From Fig. 7, it can be seen that a lower outage probability can be achieved by the TWR-NOMA-NC scheme for both users, while the outage probabilities for OWR-NOMA are always high regardless of the BS's power budgets. From these results, we see that TWR-NOMA-NC outperforms the other two schemes in terms of both sum-rate and outage performance.

\section{Conclusion}

This paper proposes an efficient TWR-NOMA-NC scheme for a cTWRN with two users. With the proposed scheme, the two users are able to exchange data with the BS within three time slots. The sum-rate performance of TWR-NOMA-NC is analyzed and a closed-form expression for the sum-rate upper bound is derived. Numerical results show that the derived bound is tight in most cases, and the proposed TWR-NOMA-NC scheme outperforms conventional TDMA-based TWR-NOMA scheme and NOMA-based one-way relaying scheme OWR-NOMA in terms of sum-rate and outage performance. 


\section{References}

[1] S. Li and Y. Sun, "Power allocation for full-duplex NOMA relaying based underlay D2D communications," KSII Transactions on Internet and Information Systems, vol. 13, no. 1, pp. 16-33, 2019. Article (CrossRef Link)

[2] Y. Saito, Y. Kishiyama, A. Benjebbour, T. Nakamura, A. Li, and K. Higuchi, "Non-orthogonal multiple access (NOMA)for cellular future radio access," in Proc. of IEEE Veh. Tech. Conf. Dresden, Germany, Jun. 2013. Article (CrossRef Link)

[3] Z. Fang, J. Hu, Y. Lu and W. Ni, "Three-User Cooperative NOMA Transmission,” IEEE Wireless Communications Letters, vol. 9, no. 4, pp. 465-469, April 2020. Article (CrossRef Link)

[4] X. Yue, Y. Liu, S. Kang, A. Nallanathan, and Z. Ding, "Exploiting Full/Half-duplex user relaying in NOMA systems,” IEEE Trans. Commun., vol. 66, no. 2, pp. 560-575, Feb. 2018. Article (CrossRef Link)

[5] J-B. Kim and I-H. Lee, "Non-orthogonal multiple access in coordinated direct and relay transmission,” IEEE Commun. Lett. vol. 19, no. 11, pp.2037-2040, Nov. 2015. Article (CrossRef Link)

[6] X. Liang, Y. Wu, D. W. K. Ng, Y. Zuo, S. Jin, and H. Zhu, "Outage performance for cooperative NOMA transmission with an AF relay,” IEEE Commun. Lett., vol. 21, no. 11, pp. 2428-2431, 2017. Article (CrossRef Link)

[7] Z. Fang, S. Shen, J. Liu, W. Ni and A. Jamalipour, "New NOMA-based Two-Way Relay Networks,” IEEE Transactions on Vehicular Technology, Early access, Nov. 2020. Article (CrossRef Link)

[8] W. Zhao, R. She and H. Bao, "Security energy efficiency maximization for two-way relay assisted cognitive radio NOMA network with self-interference harvesting," IEEE Access, vol. 7, pp. 74401-74411, 2019. Article (CrossRef Link)

[9] X. Yue, Y. Liu, S. Kang, A. Nallanathan, and Y. Chen, "Modeling and analysis of two-way relay non-orthogonal multiple access systems," IEEE Trans. Commun., vol. 66, no. 9, pp. 3784-3795, Sept. 2018. Article (CrossRef Link)

[10] F. Wei, T. Zhou, T. Xu and H. Hu, "Modeling and analysis of two-way relay networks: A joint mechanism using NOMA and network coding," IEEE Access, vol. 7, pp. 152679-152689, 2019. Article (CrossRef Link)

[11] X. Tang, K. An. K.Guo, S. Wang, X. Wang, J. Li, F. Zhou, "On the performance of two-way multiple relay non-orthogonal multiple access-based networks with hardware impairments," IEEE Access, vol. 7, pp. 128896-128909, 2019. Article (CrossRef Link)

[12] X. Wang, M.Jia, I. W. H. Ho, Q. Guo, and F. C. Lau, "Exploiting full-duplex two-way relay cooperative non-orthogonal multiple access,” IEEE Trans. Commun., vol. 67, no. 4, pp. 2716-2729, 2019. Article (CrossRef Link)

[13] B. Zheng, X. Wang, M. Wen, and F. Chen, "NOMA-Based multi-pair two-way relay networks with rate splitting and group decoding," IEEE Journal on Selected Areas in Communications, vol. 35, no. 10, pp. 2328-2341, Oct. 2017. Article (CrossRef Link)

[14] C. Y. Ho and C. Y. Leow, "Cooperative non-orthogonal multiple access with physical layer network coding," IEEE Access, vol. 7, pp. 44894-44902, 2019. Article (CrossRef Link)

[15] C. Zhang and X. Jia, "Joint beamforming optimization for NOMA-based wireless powered multi-pair two-way AF and DF relaying networks," IET Communications, vol. 13, no. 4, pp. 387-395, March 2019. Article (CrossRef Link)

[16] S. Silva, G. A. A. Baduge, M. Ardakani, and C. Tellambura, "NOMA-Aided multi-way massive MIMO relay,” IEEE Transactions on Communications, vol. 68, no. 7 pp. 4050-4062, 2020. Article (CrossRef Link)

[17] X. Li, Q. Wang, Y. Liu, T. A. Tsiftsis, Z. Ding and A. Nallanathan, "UAV-Aided multi-way NOMA networks with residual hardware impairments," IEEE Wireless Communications Letters, vol. 9, no. 9, pp. 1538 - 1542, 2020. Article (CrossRef Link). 
[18] Z. Ding, I. Krikidis, J. Thompson, and K. K. Leung, "Physical layer network coding and precoding for the two-way relay channel in cellular systems,” IEEE Trans. Signal Process, vol. 59, no. 2, pp. 696-712, 2011. Article (CrossRef Link)

[19] C. Sun, C. Yang, Y. Li, and B. Vucetic, "Transceiver design for multiuser multi-antenna two-way relay cellular systems,” IEEE Trans. Commun., vol. 60, no. 10, pp.2893-2903, Oct. 2012. Article (CrossRef Link)

[20] E. Chiu and V. K. N. Lau, "Cellular multiuser two-way MIMO AF relaying via signal space alignment: Minimum weighted SINR maximization,” IEEE Trans. Signal Process., vol. 60, pp. 4864-4873, Sep. 2012. Article (CrossRef Link)

[21] H. J. Yang, Y. Choi, N. Lee et al., “Achievable sum-rate of MU-MIMO cellular two-way relay channels: Lattice code-aided linear precoding,” IEEE J. Selec. Areas Commun., vol. 30, no. 8, pp. 1304-1318, 2012. Article (CrossRef Link)

[22] M. Gan, Z. Ding, and X. Dai, "Application of analog network coding to MIMO two-way relay channel in cellular systems,” IEEE Trans. Signal Process., vol. 20, no.7, pp. 641-644, July 2013. Article (CrossRef Link)

[23] Z. Fang, Y. Lu, J. Shi, L.Jin, and J. Ji, “A non-orthogonal multiple Access based relaying scheme for cellular two-way relay networks,” in Proc. of ICISS 2019, Tokyo, Japan, pp. 239-242, 2019. Article (CrossRef Link)

[24] D. Do, A. Le and B. M. Lee, "NOMA in cooperative underlay cognitive radio networks under imperfect SIC,” IEEE Access, vol. 8, pp. 86180-86195, 2020. Article (CrossRef Link)

[25] M. Abramowitz, and I. A. Stegun, "Handbook of Mathematical Functions with Formulas, Graphs, and Mathematical Tables,” American Journal of Physics, vol. 56, no. 10, p. 958, 1988. Article (CrossRef Link)

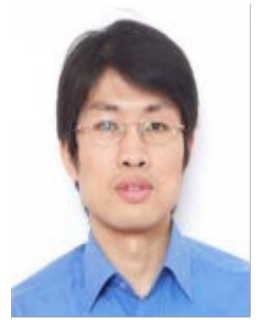

Guosheng Li received the B. E. degree in computer science from Hubei University of Technology in 2000, and the M. S. degree in software engineering from Hangzhou Dianzi University in 2004. He is a Lecture in the School of Intelligent Information Engineering, Zhejiang Wanli University, Ningbo, China. His research interests include mobile communications, embedded systems and mobile applications developing. 